\title{
ON THE PURELY INSEPARABLE CLOSURE OF RINGS
}

\author{
SHIZUKA SATO
}

\begin{abstract}
Let $K \subseteq R$ be commutative rings with identity 1 . Let $D=\left\{D_{i}\right\}$ be a higher derivation of $R$. We shall prove in this paper that if $K$ is invariant with respect to $D$, the purely inseparable closure $\overline{K_{R}}$ of $K$ in $R$ is invariant with respect to $D$ and the formal power series ring $\overline{K_{R}}[[t]]$ is purely inseparably closed in $R[[t]]$.
\end{abstract}

1. Introduction. Let $K \subseteq R$ be commutative rings with identity 1 . Let $D=\left\{D_{i}\right\}$ be a higher derivation of $R$. We shall write $D(K) \subseteq K$ if $D_{i}(K) \subseteq K$ for all $i$. We shall call $R$ purely inseparable over $K$ if for every element $x$ of $R$ there is an integer $n$ such that $(x \otimes 1-1 \otimes x)^{n}=0$ in $R \otimes_{K} R$ (cf. [1]). It is immediately proved that there exists the maximum purely inseparable subalgebra over $K$ contained in $R$. We shall call this subalgebra the purely inseparable closure of $K$ in $R$ and we shall write $\overline{K_{R}}$. We shall prove that $D(K) \subseteq K$ implies $D\left(\overline{K_{R}}\right) \subseteq \overline{K_{R}}$ and the formal power series ring $\overline{K_{R}}[[t]]$ is purely inseparably closed in $R[[t]]$.

2. The purely inseparable closure. Let $K \subseteq R$ be commutative rings with identity 1 . Let $\left\{K_{\lambda}\right\}_{\lambda \in \Lambda}$ be the set of purely inseparable subalgebras over $K$ contained in $R$ and let $\bar{K}_{R}$ be the subalgebra of $R$ generated by $\left\{K_{\lambda}\right\}_{\lambda \in \Lambda}$ over $K$. Then it is evident that $\overline{K_{R}}$ is the maximum purely inseparable subalgebra over $K$ in $R$. We shall call $\overline{K_{R}}$ the purely inseparable closure of $K$ in $R$. If $\overline{K_{R}}=K$, we shall call $K$ purely inseparably closed in $R$.

EXAMPLE. Let $K$ be a field of characteristic $p>0$ and let $X, Y$, and $Z$ be three indeterminates over $K$. Let $R=K[X, Y, Z] /\left(Y^{p}-X\right)=K[x, y, z]$. Then $R$ is a domain and $K[x, y]$ is purely inseparable over $K[x]$. Assume $S$ is a purely inseparable subalgebra over $K[x]$ containing $K[x, y]$. An element $f \in S$ can be written in the form

$$
f=\sum_{i=0}^{n} g_{i}(x, y) z^{i}, \quad g_{i}(x, y) \in K[x, y] .
$$

Then we have $f^{p^{m}} \otimes 1-1 \otimes f^{p^{m}}=0$ for some integer $m$ in $S \otimes_{K[x]} S$. Since $g_{i}(x, y)^{p^{m}} \in K[x]$, we have

$$
\sum_{i=1}^{n} g_{i}(x, y)^{p^{m}}\left(z^{i p^{m}} \otimes 1-1 \otimes z^{i p^{m}}\right)=0
$$

and hence

$$
g_{i}(x, y)^{p^{m}}=0 \quad \text { for all } i \geq 1 .
$$

Received by the editors June 4, 1986.

1980 Mathematics Subject Classification (1985 Revision). Primary 13B02; Secondary 13B10.

Key words and phrases. Purely inseparable algebras, higher derivations. 
Since $R$ is a domain $\mathrm{D}$, we have $g_{i}(x, y)=0$ for all $i$ and $f=g_{0}(x, y)$. Therefore we have $S \subseteq K[x, y]$ and it follows that the purely inseparable closure $\overline{K[x]_{R}}$ of $K[x]$ in $R$ is $K[x, y]$.

A higher derivation $D$ of $R$ is an infinite sequence $D=\left\{D_{0}, D_{1}, D_{2}, \ldots\right\}$ of mappings $D_{i}$ of $R$ into $R$ such that

$$
\begin{aligned}
& D_{i}(x+y)=D_{i}(x)+D_{i}(y), \\
& D_{i}(x y)=\sum_{j=0}^{i} D_{j}(x) D_{i-j}(y) \quad \text { for } x, y \in R, \\
& D_{0}(1)=1 .
\end{aligned}
$$

Let $D=\left\{D_{i}\right\}$ be a higher derivation of $R$ and let $e^{t D}=D_{0}+t D_{1}+t^{2} D_{2}+\cdots(t$ is a variable). Then $e^{t D}$ is a homomorphism of $R$ into $R[[t]]$.

LEMMA 1. Let $K \subseteq S \subseteq R$ be commutative rings with identity 1 . Let $D=\left\{D_{i}\right\}$ be a higher derivation of $R$. Assume $S$ is a subalgebra of $R$ purely inseparable over $K$. If $D(K) \subseteq K$, then $e^{t D}(S)$ is a purely inseparable subalgebra of $R[[t]]$ over $e^{t D}(K)$.

PROOF. Let $L$ be a reduced $e^{t D}(K)$-algebra and let $\psi, \phi$ be $e^{t D}(K)$-algebra homomorphisms of $e^{t D}(S)$ into $L$ such that $\psi i=\phi i$ where $i$ is the canonical injection of $e^{t D}(K)$ into $e^{t D}(S)$. We shall consider the commutative diagram:

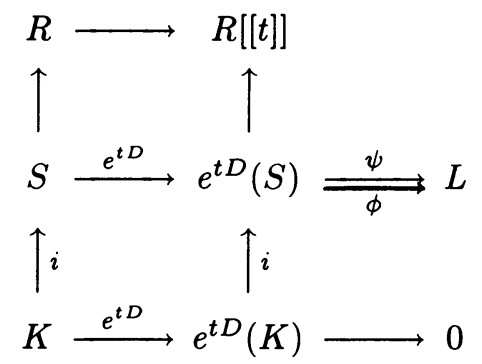

Then, since $L$ is regarded as a $K$-algebra by $e^{t D}$, we have $\psi i e^{t D}=\phi i e^{t D}$. Hence we have $\psi i e^{t D}=\psi e^{t D} i$ and $\phi i e^{t D}=\phi e^{t D} i$. Since $S$ is purely inseparable over $K$, it holds that $\psi e^{t D}=\phi e^{t D}$ and hence $\psi=\phi$. Therefore $e^{t D}(S)$ is purely inseparable over $e^{t D}(K)$.

LEMMA 2. Using the same terminology as in Lemma 1, let $T$ be an intermediate ring as $K \subseteq T \subseteq R$. Then if $S$ is a subalgebra of $R$ purely inseparable over $K$ then $S T$ is purely inseparable over $T$.

PROOF. Let $U$ be a reduced $T$-algebra and let $\psi, \phi$ be $T$-algebra homomorphisms of $S T$ into $U$ satisfying $\psi i=\phi i$. We shall consider the commutative diagram:

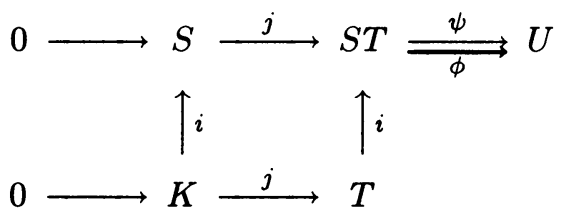

$(i, j$ are the canonical injections). From $\psi i=\phi i$ we have $\psi i j=\phi i j$ and hence $\psi j i=\phi j i$. Since $S$ is purely inseparable over $K$, it holds that $\psi=\phi$ and therefore $S T$ is purely inseparable over $T$. 
LEMMA 3. Let $K \cong R$ be commutative rings with identity 1 and let $f$ be a mapping of the product space $R[[t]] \times R[[t]]$ into $\left(R \otimes_{K} R\right)[[t]]$ ( $t$ is a variable) such that

$$
f\left(\sum a_{i} t^{i}, \sum b_{i} t^{i}\right)=\sum_{k=0}^{\infty} \sum_{i+j=k}\left(a_{i} \otimes b_{j}\right) t^{k}, \quad a_{i}, b_{j} \in R .
$$

Then $f$ is bilinear and hence we have a linear mapping $\bar{f}$ of $R[[t]] \otimes_{K[[t]]} R[[t]]$ into $\left(R \otimes_{K} R\right)[[t]]$. By a simple calculation, this mapping is a ring homomorphism.

THEOREM 1. Let $K \subseteq R$ be commutative rings with identity 1 and let $D=\left\{D_{i}\right\}$ be a higher derivation of $R$. If $D(K) \subseteq K$, we have $D\left(\overline{K_{R}}\right) \subseteq \overline{K_{R}}$.

PROOF. Let $S$ be the subalgbra of $R$ generated by $\left\{D_{i}(a)\right\}_{a \in \overline{K_{R}}, i}$ over $\overline{K_{R}}$. By Lemmas 1 and $2, e^{t D}\left(\overline{K_{R}}\right) K[[t]]$ is purely inseparable over $e^{t D}(K) K[[t]]=K[[t]]$. Therefore, for any element $a \in \overline{K_{R}}$, there is an integer $n$ such that

$$
\left(e^{t D}(a) \otimes 1-1 \otimes e^{t D}(a)\right)^{n}=0
$$

in

$$
e^{t D}\left(\overline{K_{R}}\right) K[[t]] \otimes K[[t]] e^{t D}\left(\overline{K_{R}}\right) K[[t]] .
$$

Since $e^{t D}\left(\overline{K_{R}}\right) K[[t]] \subseteq S[[t]]$, we have $\left.e^{t D}(a) \otimes 1-1 \otimes e^{t D}(a)\right)^{n}=0$ in $S[[t]] \otimes_{K}[[t]]$ $S[[t]]$ and hence

$$
\bar{f}\left(e^{t D}(a) \otimes 1-1 \otimes e^{t D}(a)\right)^{n}=0
$$

in $\left(S \otimes_{K} S\right)[[t]]$ by Lemma 3 . By a simple calculation we have

$$
\left[e^{t D}(a) \otimes 1-1 \otimes e^{t D}(a)-\sum_{i=0}^{m-1}\left(D_{i}(a) \otimes 1-1 \otimes D_{i}(a)\right) t^{i}\right]^{n 2^{m}}=0
$$

in $\left(S \otimes_{K} S\right)[[t]]$ and hence $\left(D_{m}(a) \otimes 1-1 \otimes D_{m}(a)\right)^{n 2^{m}}=0$ in $S \otimes_{K} S$. Since $S$ is generated by $\left\{D_{i}(a)\right\}_{0 \leq i<\infty, a \in \overline{K_{R}}}$ over $\overline{K_{R}}, S$ is purely inseparable over $K$. Therefore we have $\overline{K_{R}}=S$ and $D\left(\overline{K_{R}}\right) \subseteq \overline{K_{R}}$.

THEOREM 2. Let $K \subseteq R$ be commutative rings with identity 1 . Then $\overline{K_{R}}[[t]]$ is purely inseparably closed in $R[[t]]$.

ProOF. Let $D=\left\{D_{k}\right\}$ be a sequence of mappings of $R[[t]]$ into $R[[t]]$ such that $D_{k}\left(\sum a_{i} t^{i}\right)=a_{k} t^{k}$ for $\sum a_{i} t^{i} \in R[[t]], k=0,1,2,3, \ldots$ Then $D$ is a higher derivation of $R[[t]]$ such that $D\left(\overline{K_{R}}[[t]]\right) \subseteq \overline{K_{R}}[[t]]$.

Let $T$ be the purely inseparable closure of $\overline{K_{R}}[[t]]$ in $R[[t]]$ and let $\alpha=\sum a_{i} t^{i} \in T$. By Theorem 1 we have $D_{k}(\alpha) \in T$ and hence $\left(a_{k} \otimes 1-1 \otimes a_{k}\right)^{n}\left(t^{n k} \otimes 1\right)=0$ in $T \otimes_{K_{R}[[t]]} T$ for some integer $n$. Let $S$ be a subring of $R$ generated by coefficients

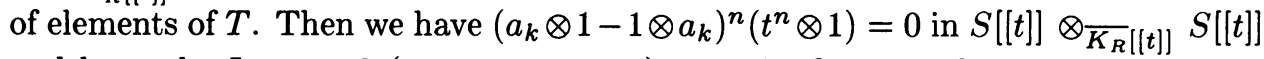
and hence by Lemma $3\left(a_{k} \otimes 1-1 \otimes a_{k}\right)^{n}=0$ in $S \otimes_{\overline{K_{R}}} S$. By the definition of $S, S$ is purely inseparable over $\overline{K_{R}}$. Since $\overline{K_{R}}$ is purely inseparably closed in $R$, we have $\overline{K_{R}}=S$. Therefore it follows $T=\overline{K_{R}}[[t]]$ and hence $\overline{K_{R}}[[t]]$ is purely inseparably closed in $R[[t]]$. 


\section{REFERENCES}

1. S. Sato, On purely inseparable algebras and P.H.D. rings, Trans. Amer. Math. Soc. 266 (1981), 483-498.

FACUlty of Engineering, Oita University, Oita 870-11, JAPAN 